\title{
Zur Kenntnis der in den Nahrungsmitteln enthaltenen Enzyme und ihrer Mitwirkung bei der Verdauung.
}

\author{
Von
}

Arthur Scheunert und Walther Grimmer.

(Aus der physiologisch-chemischen Abteilung der kgl. sächs. tierärztlichen Hochschule zu Dresden.)

(Geh. Med.-Rat Prof. Dr. Ellenberger.)

(Der Redaktion zugegangen am 29. März 1906.)

Durch die Untersuchungen Ellenbergers und seiner Mitarbeiter über die Verdauung der Einhufer und der Wiederkäuer war seinerzeit festgestellt worden, daß im Magen dieser Tiere eine sehr bedeutende Amylolyse stattfindet, indem die in den Nahrungsmitteln enthaltene Stärke in lösliche Abbauprodukte, Dextrin, Maltose, usw. umgewandelt wird, die dann teilweise weiter in Milchsäure gespalten werden.

So werden im Magen des Pferdes bei Haferfütterung von der in diesem Futtermittel enthaltenen Stärke bis zu 60\%, bei Maisfütterung bis zu $50 \%{ }^{2}$ ) und mehr verdaut. Im Mageninhalte finden sich ganz erhebliche Mengen löslicher, Fehlingsche Lösung reduzierender Abbauprodukte der Stärke und freie Milchsäure. So konnten im Mageninhalte bei Haferfütterung teilweise 50, ja sogar $120 \mathrm{~g}$ Zucker neben $10-50 \mathrm{~g}$ Milchsäure, bei Maisfütterung (l. c.) bis zu $60 \mathrm{~g}$ Zucker nachgewiesen werden. Da im Hafer oft gar kein, im Mais nur eine minimale Menge $(0,26 \%$, vgl. S. 32) Zucker vorhanden ist, und da außerdem, wie wir nachgewiesen haben, im Magen fortwährend eine Resorption der Abbauprodukte der Stärke, (des Zuckers und der Milchsäure) stattfindet, so sind die erwähnten, im Mageninhalte

1) Goldschmidt, Diese Zeitschrift.

Scheunert, Pflügers Archiv, Bd. CIX, S. 145.

2) Scheunert und Grimmer, Diese Zeitschrift, Bd. XLVII, S. 88. 
auftretenden Mengen dieser Verdauungsprodukte als recht beträchtliche $z u$ bezeichnen.

Als Ursachen für die im Magen der Einhufer und Wiederkäuer ablaufende Stärkeverdauung kamen anfangs nach den umfangreichen Ellen berge rschen Untersuchungen zunächst nur das von den Speicheldrüsen produzierte Ptyalin und das in der Magenschleimhaut enthaltene, von gewissen Magendrüsen produzierte amylolytische Enzym in Betracht. Da aber, wie zahlreiche in unserem Institute durchgeführte Versuche dargetan haben, durch diese beiden in ungenügender Menge und ungenügender Wirksamkeit vorhandenen Enzyme die beträchtliche, schon nach kurzer Zeit im Magen herrschende Stärkeverdauung nicht erklärt werden kann, ließ Ellenberger weitere Untersuchungen zur Auffindung anderer Fermentquellen ausführen. Bei dieser Gelegenheit wurde von Goldschmidt ${ }^{1}$ ) das Vorkommen eines auf Stärke saccharifizierend wirkenden Pilzes in der Luft festgestellt, der bei der Nahrungsaufnahme mit in den Magen eintreten kann, während alle Versuche, die das Vorkommen von anderen bei der Magenverdauung mitwirkenden diastatischen Enzymen im Tierkörper (Untersuchung der Extrakte der cytoblastischen Gewebe der Mund- und Rachenhöhle und der Speiseröhre, der Tonsillen, der Drüsen der Rachenhöhle, des Schlundkopfes und des Oesophagus) negative Resultate hatten. Die endgültige Lösung der Frage wurde aber erst dadurch herbeigeführt, daß Ellenberger die Nahrungsmittel untersuchte. Zur Untersuchung gelangte zunächst Hafer, der in einer Mischung mit 60 bis $70 \%$ Wasser bei Bluttemperatur in den Brutschrank eingestellt wurde. Die Analyse der abgepreßten Flüssigkeit ergab, daß im Digestionsgemisch eine lebhafte, mit der Zunahme der Digestionsdauer wachsende Zuckerbildung eingetreten war.

War aber der Hafer vorher auf $100^{\circ}$ erhitzt worden, so konnte eine Zunahme der etwa im Hafer bereits vorhandenen Zuckermenge mit der Dauer des Aufenthaltes im Thermostaten, also ein amylolytischer Vorgang, nicht beobachtet werden.

Hierdurch kam Ellenberger ${ }^{2}$ ) auf die Vermutung, daß

1) Goldschmidt, Diese Zeitschrift, Bd. X, S. 299.

2) Archiv f. wissenschaftl. u. prakt. Tierheilkunde, Bd. XIII, S. 188. 
im Hafer selbst ein Enzym vorhanden sein müsse, welches unter den im Magen gegebenen Bedingungen eine amylolytische Wirkung auf das Stärkemehl des Hafers zu entfalten vermag. Ein weiterer Beweis für die Richtigkeit dieser Annahme konnte durch das Tierexperiment erbracht werden. Von 2 Pferden erhielt, nachdem der Magen durch 36 stündiges Hungern von den Resten früherer Mahlzeiten befreit worden war, das eine $2 \mathrm{~kg}$ rohen Hafer, während das andere $2 \mathrm{~kg}$ vorher 5 Minuten in siedendem Wasser erhitzten Hafer vorgelegt bekam. 2 Stunden nach Beendigung der Mahlzeit wurden die Tiere getötet und der Mageninhalt auf den Gehalt an Zucker und Säure (Milchsäure) geprüft. Während das mit rohem Hafer gefütterte Pferd einen Zuckergehalt von $1,5 \%$ und einen Säuregrad von $0,4 \%$ (als Milchsäure berechnet) im Mageninhalte aufwies, wurden bei dem mit gekochtem Hafer gefütterten Tiere nur 0,5\% Zucker und $0,1 \%$ Milchsäure gefunden. Durch diese Versuche war demnach bewiesen, daß im rohen Hafer ein durch Siedehitze zerstörbares, unter den im Magen gegebenen Bedingungen kräftig wirkendes amylolytisches Enzym vorhanden ist, und daß ein Teil, und zwar kein unbeträchtlicher, der im Magen ablaufenden Stärkeverdauung der Wirkung dieses Enzyms zugeschrieben werden muß.

Diese, auf die Ermittelung von in den Nahrungsmitteln enthaltenen, bei der Magen- und Darmverdauung wirksamen Enzymen gerichteten Untersuchungen sind im Laufe der Jahre in unserem Institute fortgesetzt, auf die verschiedensten Nahrungsmittel ausgedehnt und gelegentlich anderer Arbeiten nachgeprüft worden. So ist das Vorkommen eines bei Körpertemperatur wirksamen, amylolytiśchen Fermentes in verschiedenen Mehlsorten, sowie im Roggenstroh und Wiesenheu, ${ }^{1}$ ) und das Vorkommen eines proteolytischen und Milchsäure bildenden Enzymes im Hafer nachgewiesen worden.

Das Vorkommen von diastatischen und proteolytischen Enzymen in den Pflanzen und Pflanzensamen, besonders in keimenden Samen, ist eine schon längst bekannte und durch viele ausgezeichnete Experimentaluntersuchungen belegte Tat-

1) Veterinärber. 18. 
sache. Die darüber existierende Literatur ist sehr umfangreich, doch liegt keine Veranlassung vor, darauf näher einzugehen, da es sich bei den in unserem Institute ausgeführten Untersuchungen lediglich darum handelte, das Vorhandensein von solchen Enzymen in den pflanzlichen Nahrungsmitteln nachzuweisen, welche imstande sind, die wichtigsten Nährstoffe derselben (Kohlehydrate, Eiweißkörper und Fette) unter den Bedingungen, denen sie im Verdaungsschlauche ausgesetzt sind, zu den für den Organismus verwertbaren Produkten abzubauen. Diese Frage, welche für die Beurteilung der Verdauungsvorgänge besonders der Herbi- und der Omnivoren von der weitgehendsten Bedeutung ist, sind in der oben erwähnten Literatur kaum erwähnt worden.

Durch die eingangs geschilderten Untersuchungen von Ellenberger und seinem Mitarbeiter V. Hofmeister ist zum erstenmal auf die große Rolle, die die Nahrungsmittelenzyme bei der Verdauung spielen, hingewiesen worden. Bei der Fortsetzung derartiger Versuche, die in unserem Institute auf einige weitere, teilweise als Körnerfutter dienende Futtermittel ausgedehnt wurden, haben sich einige neue Tatsachen ergeben, die im folgenden mitgeteilt werden sollen. Die neuen Versuche erstreckten sich auf Hafer, Mais, Pferdebohnen, Lupinenkörner, Buchweizen und Wicken und bezweckten, das Vorkommen und die Wirkung eines amylolytischen, proteolytischen, Milchsäure bildenden und cytohydrolytischen Enzyms unter den im Magen und Darm während der Verdauung herrschenden Verhältnissen festzustellen.

\section{Untersuchung über das zuckerbildende Nahrungsmittelenzym.}

Zunächst wurde in jedem Nahrungsmittel der in ihm enthaltene, lösliche Zucker bestimmt, indem je $5 \mathrm{~g}$ der Nahrungsmittel mit der 10 fachen Menge eiskalten Wassers übergossen wurden und nach gründlichem Vermengen eine Viertelstunde im Eisschrank sich selbst überlassen blieben. Im Filtrat erfolgte dann nach der weiter unten beschriebenen Methode die Bestimmung des gelösten Zuckers. Zur Untersuchung auf das Enzym gelangten die Nahrungsmittel, in denselben Mengen- 
verhältnissen mit Wasser gemengt, auf 2 und 6 Stunden bei $39^{\circ}-40^{\circ}$ in den Brütofen. Für jede Verdauungszeit setzten wir stets eine besondere Portion in einem besonderen Erlenmeyerkölbchen an, da sich herausstellte, daß bei Entnahme der Proben für die verschiedenen Verdauungsstunden aus demselben Kölbchen infolge der dadurch hervorgerufenen Flüssigkeitsverminderung ungenaue Resultate erhalten wurden. Da es ferner von Interesse schien, die Wirkung des Enzyms nicht nur bei neutraler Reaktion, die im Mageninhalte wenigstens gleichzeitig an allen Stellen niemals herrscht, kennen zu lernen, führten wir die Verdauungsversuche auch noch bei alkalischer, entsprechend der rein amylolytischen Periode der Magenverdauung $\left(0,2 \% \mathrm{Na}_{2} \mathrm{CO}_{3}\right)$ und schwach salzsaurer, entsprechend der späteren gemischt amylolytisch-proteolytischen und der vorwiegend proteolytischen Periode Reaktion $(0,2 \% \mathrm{HCl})$ aus.

Zur Bestimmung des Zuckers versetzten wir nach Ellenberger das Gemisch unter Umschütteln zunächst mit $10 \mathrm{ccm}$ Phosphorwolframsäurelösung und $10 \mathrm{ccm}$ einer Salzsäure vom spezifischen Gewicht 1,125, um die in Lösung befindlichen Eiweißkörper (mit Einschluß von Albumosen, Proteosen, Peptonen usw.), deren Anwesenheit die quantitative Zuckerbestimmung stört, zu entfernen. Nach einiger Zeit setzt sich bei dieser Behandlung der Niederschlag zu Boden. Hierauf wird die überstehende Flüssigkeit abfiltriert, und der Filtrierrückstand mit Wasser solange ausgewaschen, bis im Filtrat eine Zuckerreaktion nicht mehr erhalten werden kann. Das Filtrat wird hierauf mit Natronlauge alkalisiert, der eventuell entstehende Phosphorwolframsäureniederschlag abfiltriert und das so erhaltene klare und schwach alkalische Filtrat auf $200 \mathrm{ccm}$ gebracht. In je $20 \mathrm{ccm}$ dieses Filtrates wird der Zucker nach der Allihnschen Methode bestimmt und als Dextrose in Rechnung gebracht. Die Resultate unserer Versuche sind in der folgenden Tabelle zusammengestellt.

Wie aus der Tabelle hervorgeht und auch schon früher in unserem Institute nachgewiesen worden ist, ist der Zuckergehalt der Nahrungsmittel sehr verschieden. Während wir im Buchweizen gar keinen oder nur Spuren von mit Wasser extrahier- 
barem Zucker vorfanden, fanden sich in den Lupinen 3,4\% vor. Ähnliche Unterschiede ergaben schon die früher ausgeführten Versuche, bei denen im Erbsenmehl nur Spuren, in Kartoffeln, Reis und Stroh ca. 0,1\%, in Gerste, Roggen, Hafer, Mais ca. 0,2-0,5\% und im Wiesenheu 2\% Zucker gefunden worden waren. Es mag hierzu noch bemerkt werden, daß der Zuckergehalt jedes Futtermittels für sich auch verschieden ist, je nach dem Alter, der Art der Aufbewahrung usw., man findet also Hafer, der zuckerfrei ist, solchen der $0,2 \%$, anderen, der $0,5 \%$ Zucker enthält usw. Es muß also jedes zu einem Versuche verwendete Futtermittel in jedem Falle analysiert werden.

\begin{tabular}{|c|c|c|c|c|c|}
\hline \multirow[t]{2}{*}{$\begin{array}{c}\text { Nahrungs } \\
\text { mittel }\end{array}$} & \multirow{2}{*}{$\begin{array}{l}\text { Zucker in } \\
\text { Prozenten } \\
\text { zu Beginn } \\
\text { des } \\
\text { Versuchs }\end{array}$} & \multirow{2}{*}{$\begin{array}{l}\text { Dauer des } \\
\text { Aufent- } \\
\text { haltes } \\
\text { im } \\
\text { Brutofen }\end{array}$} & \multicolumn{3}{|c|}{$\begin{array}{l}\text { Zucker im Mittel in Prozenten während } \\
\text { des Versuches. } \\
\text { Die Reaktion der Verdauungsflüssigkeit } \\
\text { ist beim Versuchsbeginn: }\end{array}$} \\
\hline & & & $\begin{array}{c}\text { sauer } \\
0,2 \% \mathrm{HCl}\end{array}$ & neutral & $\begin{array}{c}\text { alkalisch } \\
0,2 \% \mathrm{Na}_{8} \mathrm{CO}_{8}\end{array}$ \\
\hline \multirow{4}{*}{ Mais } & \multirow{4}{*}{0,26} & 2 & 0 & 1,27 & 1,52 \\
\hline & & 4 & - & 2,07 & - \\
\hline & & 6 & Spuren & 2,77 & 2,88 \\
\hline & & 8 & - & 3,31 & - \\
\hline \multirow{2}{*}{ Hafer } & \multirow{2}{*}{0,81} & 2 & 0 & - & 1,32 \\
\hline & & 6 & Spuren & - & 2,20 \\
\hline \multirow{2}{*}{$\begin{array}{l}\text { Pferde- } \\
\text { bohnen }\end{array}$} & \multirow{2}{*}{1,6} & 2 & 1,03 & 3,03 & 4,13 \\
\hline & & 6 & 3,6 & 6,6 & 5,4 \\
\hline \multirow{2}{*}{ Lupinen } & \multirow{2}{*}{3,38} & 2 & 2,58 & 3,84 & 3.60 \\
\hline & & 6 & 4,6 & 9,4 & 8,35 \\
\hline \multirow{2}{*}{$\begin{array}{l}\text { Buch- } \\
\text { weizen }\end{array}$} & \multirow{2}{*}{0} & 2 & 0 & 0 & 0,69 \\
\hline & & 6 & Spuren & Spuren & 2,10 \\
\hline \multirow{2}{*}{ Wicke } & \multirow{2}{*}{2,1} & 2 & 1,55 & 3,03 & 2,03 \\
\hline & & 6 & 3,2 & 7,5 & 10,5 \\
\hline
\end{tabular}

Bei den neu untersuchten Futtermitteln nahm, wie bei den früher untersuchten, bei den bei Bluttemperatur angestellten Verdauungsversuchen die Zuckermenge im Verdauungsgemisch von Stunde zu Stunde zu. So ist im Maximum unter den günstigsten Bedingungen nach 6 stündiger Verdauungszeit der 
Zuckergehalt bei Mais auf das 10 fache, bei Wicken um das 5 fache, bei Pferdebohnen um das 4 fache, bei Lupinen um das 3 fache der ursprünglich in den rohen Früchten enthaltenen Zuckermenge gestiegen. Die Erklärung dieser Tatsache kann nur in der Wirkung eines amylolytischen Enzyms gefunden werden, welches in den rohen Früchten enthalten ist und seine Wirkung unter den gegebenen Versuchsbedingungen auf das Stärkemehl derselben entfaltet.

Besonders bemerkenswert ist, daß wir auch jetzt wieder feststellen konnten, daß die Nahrungsmittel, die den reichlichsten Gehalt an Zucker aufweisen, auch beim Verdauungsversuch die größten Zuckermengen zu bilden vermögen. Am zuckerärmsten ist der Buchweizen, der überhaupt keinen Zucker enthält, in ihm hat sich nach 6 Stunden in alkalischer Lösung erst 2\% Zueker gebildet, Wicken und Lupinenkörner, mit dem größten Zuckergehalt, haben nach gleicher Zeit die größte Menge $(8-10 \%$ ) gebildet.

Von besonderem Interesse erscheint ferner die Betrachtung der Wirkungsweise des saccharifizierenden Nahrungsmittelenzyms bei verschiedener Reaktion des Verdaungsgemisches. Wir wählten, wie oben erwähnt, ähnliche Konzentrationen von Säure und Alkali, wie sie im Magen- und Darminhalt der Pflanzenfresser während der Verdauung zu herrschen pflegen, und können auf Grund der vorstehenden Tabelle feststellen, daß das amylolytische Enzym aller von uns untersuchten. Nahrungsmittel bei schwach alkalischer und mit Ausnahme des Buchweizens anch bei neutraler Reaktion eine saccharifizierende Wirkung zu ontfalten vermag. Je nach der Art der Nahrungsmittel und der Dauer der Verdauung wird diese Wirkung durch alkalische oder neutrale Reaktion begünstigt. Auf alle Fälle kann das Enzym unter den in unserem Institute zuerst erkannten Bedingungen, die während der ersten und im Anfange der zweiten Periode der Magenverdauung der Herbi- und Omnivoren bei Aufnahme einer stärkehaltigen Nahrung im. Magen herrschen, eine kräftige Stärkeverdauung bewirken.

Bemerkenswert scheinen uns besonders die Resultate zu sein, die wir bei den Verdauungsversuchen bei schwach saurer 
Reaktion erhalten haben. Das im Hafer, Mais und Buchweizen enthaltene amylolytische Enzym wird offenbar durch eine Konzentration von $0,2 \% \mathrm{HCl}$ wirkungslos gemacht, da sich auch nach längerem Aufenthalte im Thermostaten nur Spuren von Zucker im Verdauungsgemisch nachweisen ließen. Das amylolytische Enzym der Pferdebohnen, Lupinenkörner und der Wicke hingegen verliert hierbei seine Wirksamkeit nicht, sondern behält dieselbe, wenn auch in geringerem Maße, bei. Da uns diese Eigenschaft des Enzyms, auch in saurer Lösung wirksam zu sein, sehr merkwürdig erschien, haben wir mehrfache Kontrollversuche angestellt, die bei den betreffenden Nahrungsmitteln unsere Ergebnisse bestätigten.

Für den Ablauf der Magenverdauung der Pflanzenfresser dürfte dieser Befund von großer Bedeutung sein. So haben z. B. beim Pferde die Untersuchungen von Ellenberger und Hofmeister ${ }^{1}$ ) dargetan, daß das für die Stärkeverdauung im Pferdemagen neben dem Nahrungsmittelenzym wichtigste Enzym, das Ptyalin des Pferdespeichels, bei einer Konzentration von $0,02 \% \mathrm{HCl}$ in seiner Wirkung stark beeinträchtigt und durch höhere Konzentrationen, 0,03-0,04\% $\% \mathrm{HCl}$, sogar ganz wirkungslos wird. Bei Hafer- und Maisfütterung wird also die Stärkeverdauung an den Stellen des Mageninhaltes unterbrochen werden, an denen eine solche Salzsäurekonzentration auftritt, was auch, wie in unserem Institute nachgewiesen worden ist, bekanntlich im Verlaufe der 3. Periode der Magenverdauung in der Fundusdrüsenregion der Fall ist. Gibt man hingegen ein Nahrungsmittel, dessen amylolytisches Enzym noch bei einer Konzentration von $0,2 \% \mathrm{HCl}$ wirksam ist, so wird die Stärkeverdauung auch in den späteren Verdauungsstunden, in denen bei den Herbivoren im höchsten Falle eine Konzentration von $0,2 \% \mathrm{HCl}$ und nur selten von $0,3 \%$ im Magen $\mathrm{zu}$ finden ist, erhalten. Die Beobachtung dieser Verhältnisse dürfte auch für die Wahl der Futtermittel bei Magenerkrankungen von Bedeutung sein.

Es muß weiterhin aber noch darauf aufmerksam gemacht werden, daß im Magen der Pflanzenfresser und der Pflanzennahrung aufnehmenden Omnivoren der im Mageninhalte vor-

1) Archiv f. wissenschaftl. u. prakt. Tierheilkunde, Bd. XII, S. 339. 
handene Säuregrad nur zu einem geringen Teil von Salzsăure, zu einem erheblichen Teile jedoch von Milchsäure herrührt. Diese organische Säure wirkt aber, wie diesbezügliche von uns angestellte Versuche zeigen, nicht in dem gleichen Grade wie die Salzsäure hemmend und hindernd auf die diastatische Wirkung des amylolytischen Nahrungsmittelenzymes (z. B. in Hafer und Mais) ein. Bei den Verdauungsversuchen in vitro wirkte dieses Enzym immer noch saccharifizierend, trotzdem infolge der daneben ablaufenden Milchsäuregärung sich die Säure derart ansammelte, daß sich im Verdauungsgemische 0,3-0,4.\% Milchsäure vorfanden.

Auch die mit Zusatz von Milchsäure angestellten Versuche haben gezeigt, daß die Milchsäure in Konzentrationen von $0,5 \%$ und darüber das Enzym in seiner Wirkung nicht behindert.

Es geht aus allem diesen hervor, daß das amylolytische Nahrungsmittelenzym des Hafers, des Maises etc., dessen Wirkung durch 0,1- und 0,2\%ige Salzsäure aufgehoben wird, sehr wohl noch saccharifizierend im Magen wirken kann, trotzdem der Mageninhalt einen Säuregrad von $0,2 \%$ und darüber zeigt, falls dieser Säuregrad nicht durch das Vorhandensein von freier Salzsäure hervorgerufen wird, bzw. falls diese Säure neben der Milchsäure nicht in genügend hoher, eine Hemmung der amylolytischen Wirkung verursachender Konzentration vorhanden ist. Diese Tatsache ist von großer Bedeutung für die Beurteilung der Magenverdauung der Herbi- und Omnivoren.

Bei der Betrachtung der bei den Verdauungsversuchen in salzsaurer Lösung gewonnenen Prozentzahlen fällt auf, daß bei sämtlichen Nahrungsmitteln nach 2 Stunden weniger Zucker im Verdauungsgemisch vorhanden ist, als darin überhaupt bei viertelstündiger Extraktion im Eisschranke nachgewiesen werden konnte. Wir: glaubten zuerst, daß unsre Methode der Bestimmung des Zuckergehaltes der Nahrungsmittel insofern nicht einwandfrei sei, als vielleicht bei neutraler Reaktion schon im Eisschrank durch Enzymwirkung eine Vermehrung ihres Zuckergehaltes veranlaßt werde. Es wurde deshalb ein Kontrollversuch ausgeführt, bei dem wir, um Enzymwirkung auszuschließen, zur Extraktion eine 1\% 10 ige 
eisgekühlte Salzsäure verwandten und alle Manipulationen ausschließlich im Eisschranke vornahmen. Die Zuckerbestimmung ergab jedoch genau denselben Wert wie vorher. Eine Erklärung für die erwähnte Tatsache kann demnach nur darin gefunden werden, daß infolge der salzsauren Reaktion irgendwelche Vorgänge im Verdauungsgemisch ablaufen, die eine Zersetzung der entstehenden reduzierenden Kohlehydrate bewirken, welche wenigstens anfänglich beträchtlicher ist, als die durch die enzymatische Wirkung bedingte Zunahme derselben. Um festzustellen, ob etwa als Zersetzungsprodukte des Zuckers Säuren auftreten, stellten wir folgende Versuche mit Proben von Hafer und Pferdebohnen an. Je 2 Proben von je $5 \mathrm{~g}$ der beiden Nahrungsmittel wurden mit $50 \mathrm{ccm} 0,2 \%$ iger $\mathrm{HCl}$, deren Titer gegen $\mathrm{n} / \mathbf{1 0}-\mathrm{NaOH}$ vorher genau bestimmt worden war, in den Brütofen eingestellt. Nach einer Viertelstunde unterbrachen wir den Versuch, filtrierten $a b$ und bestimmten im Filtrat von neuem die Acidität mit $\mathrm{n} / \mathbf{1 0} \cdot \mathrm{NaOH}$. Dies erwies sich nach Vorversuchen als unbedingt nötig, da jedes Nahrungsmittel Bestandteile, die sowohl organischer wie anorganischer Natur sein dürften, enthält, welche ein gewisses Säurebindungsvermögen besitzen. Die Zeit von einer Viertelstunde wählten wir, um sicher sein zu können, daß dann alle Neutralisationsbzw. salzbildenden Vorgänge abgelaufen sein würden, die Acidität des Verdauungsgemisches eine Verminderung also nicht mehr erfahren könne. Nach 2 stündigem Aufenthalte im Thermostaten wurde die 2. Probe abfiltriert und wiederum die Acidität mit $\mathrm{n} / 10-\mathrm{NaOH}$ bestimmt. War jetzt ein Anwachsen derselben festzustellen, so könnte dieses wohl nur dadurch erklärt werden, daß Vorgänge im Verdauungsgemisch abgelaufen sind, die ein Auftreten freier Säure im Gefolge haben. Die Resultate waren folgende:

I. Hafer:

$25 \mathrm{ccm}$ neutralisieren $16 \mathrm{ccm} \mathrm{n} / 10-\mathrm{NaOH}$

25 . Lösung von $1 / 4$ Stunde im Brütofen neutralisieren $11,6 \mathrm{ccm}$ n/10-NaOH

$25,>, 2$ Standen $>, 12,1,>$

Auf Milchsăure berechnet war demnach eine Aciditătszunahme von $0,18 \%$ erfolgt. 


\section{Pferdebohnen :}

$25 \mathrm{ccm}$ HCl neutralisieren $16,0 \mathrm{ccm} \mathrm{n} / 10-\mathrm{NaOH}$

25 Lösung von $1 / 4$ Stunde im Brütofen neutralisieren $11,1 \mathrm{ccm} \mathbf{n} / 10-\mathrm{NaOH}$

25 > > 2 Stunden , > 12,3 , ,

Auf Milchsäure berechnet war hier eine Zunahme des Säuregehaltes um $0,43 \%$ erfolgt.

$\mathrm{Ob}$ durch diese Versuche eine Erklärung für das Verschwinden von reduzierenden Kohlehydraten gegeben werden kann, bleibt dahingestellt (man denke an das Auftreten saurer durch Proteolyse entstandener Verdauungsprodukte), jedenfalls erscheint uns das im Verdauungsgemische mit diesem Verschwinden Hand in Hand gehende Anwachsen des Säuregehaltes durch obige Versuche einwandfrei nachgewiesen.

Wenn aus den Ergebnissen der früheren und der neueren Versuche zu schließen ist, daß Nahrungsmittelenzyme bei der Verdauung der Stärke im Magen eine große Rolle spielen, so war es doch nötig, auch festzustellen, ob diese Enzyme bei der Darmverdauung ebenfalls wirken können.

In dieser Richtung ist von uns durch andere Versuche festgestellt worden, daß ein kleiner Teil der aufgenommenen Nahrung den Magen rasch durcheilt, ja beim Pferde tritt ein Teil der Nahrung, ohne der Magenverdauung ausgesetzt gewesen zu sein, schon während der Mahlzeit in den Darmkanal über. Es kann keinem Zweifel unterliegen, daß in diesen Teilen der aufgenommenen stärkemehlhaltigen Nahrung das Nahrungsmittelenzym auch im Darmkanal seine Wirkung entfalten kann, die Galle, der Darm- und Pankreassaft hindern diese Wirkung nicht; bei der in der ersten Zeit im Dünndarminhalt herrschenden alkalischen Reaktion wirkt das amylolytische Nahrungsenzym sicherlich gut. Später wird der Darminhalt infolge der Milchsäuregärung sauer. Freie Milchsäure beeinträchtigt aber das Nahrungsmittelenzym in seiner fermentativen Wirkung nicht.

Ein großer Teil der aufgenommenen Nahrung bleibt aber lange im Magen; das amylolytische Enzym wird dann in seiner Wirkung durch die sich ansammelnde Salzsäure mehr oder weniger gehemmt. Es ist nun die Frage, ob dieses Enzym im Darmkanale seine Wirksamkeit wieder erhält bzw. beibehält, 
wenn die Salzsäure nicht mehr zugegen ist, oder ob im Magen durch die Einwirkung der Salzsäure auf das Nahrungsmittelenzym dieses nicht nur in der Wirkung gehemmt, sondern zerstört worden ist.

In bezug auf das aus dem Tierkörper stammende amylolytische Enzym ist früher schon in unserem Institute festgestellt worden, daß die 0,03-0,2\% ige Salzsäure dieses Enzym nicht vernichtet, sondern daß dessen Wirksamkeit wiederkehrt, wenn die Konzentration der Salzsäure entsprechend gemindert wird, bzw. wenn die Salzsäure durch eine organische Säure ersetzt oder wenn das durch die Gegenwart der $\mathrm{HCl}$ wirkungslose Verdauungsgemisch alkalisiert wird. Allerdings zeigte sich, daß das betreffende Enzym (Ptyalin, amylolytisches Magenenzym), nachdem es der Wirkung einer $0,1-0,2 \%$ igen Salzsäure ausgesetzt gewesen war, schwächer wirkte als vorher. Es handelte sich nun darum, festzustellen, wie sich das Nahrungsmittelenzym in dieser Beziehung verhält, ob es also durch die Salzsäure in der genannten Konzentration zerstört, oder ob seine Wirksamkeit nur vorübergehend gehemmt wird, und wiederkehrt, sobald die $\mathrm{HCl}$ entfernt wird. Wir mußten dieses Enzym also zunächst der Wirkung einer $0,1-0,2 \%$ igen Salzsäure aussetzen, dann das Gemisch neutralisieren bzw. alkalisieren und feststellen, ob das Enzym jetzt überhaupt wieder bezw. in verstärktem Maße saccharifizierend auf Stärke wirkt.

Gleichzeitig haben wir auch ein Urteil über die Wirkungsfähigkeit der Enzyme für den Fall zu erhalten versucht, wenn sie zunächst der Wirkung von Alkali ausgesetzt werden und dann Säurezusatz erfolgt.

Wird die durch Salzsäure verursachte Hemmung der enzymatischen Wirkung durch Neutralisation oder Alkalisieren wieder aufgehoben, dann ist erwiesen, daß das in der Nahrung enthaltene, im Magen durch die $\mathrm{HCl}$ wirkungslose oder geschwächte amylolytische Enzym im Darm unter den dort herrschenden Verhältnissen (bei alkalischer oder auch nur durch Milchsäure bedingter saurer Reaktion) wieder verdauend wirken kann. Für die Tiere hat dies eine große Bedeutung, weil sie ja fast nur rohe, ungekochte Nahrung aufnehmen. Bei gestörter Absonderung des 
Pankreassaftes (Pankreasachylie) und anderen Störungen in der Absonderung der in den Darm sich ergießenden Verdauungssäfte ist die Frage, ob das amylolytische Enzym der Nahrung, des Speichels und des Magensaftes im Darm noch wirken kann, trotzdem es im Magen unwirksam geworden war, von sehr großer Bedeutung. Es bleiben dann die durch Störungen der Stärkeverdauung bedingten Folgen der Krankheit aus.

Zur Beantwortung dieser Fragen haben wir einige Versuche in folgender Weise ausgeführt. Es wurden zunächst bei saurer oder alkalischer Reaktion unter den üblichen Konzentrationsverhältnissen die Nahrungsmittel 6 Stunden im Brütofen gelassen, hierauf wurde genau neutralisiert und dann durch $\mathrm{Zu}$ fügen bekannter Mengen $\mathrm{HCl}$ - und $\mathrm{Na}_{2} \mathrm{CO}_{3}$-Lösung die der ursprünglichen entgegengesetzte Reaktion unter den üblichen Konzentrationsverhältnissen hergestellt. Nach weiterem 6 stündigen Aufenthalte im Thermostaten wurde der Zuckergehalt bestimmt. Die Versuche, die sich sehr weitläufig gestalteten, sind zu einem endgültigen Abschlusse nicht gelangt, ihre Resultate deuten darauf hin, daß in einigen Futtermitteln, und zwar in denen, deren Stärkemehl sowohl in saurer, als auch in alkalischer Lösung verzuckert wird, z. B. Pferdebohnen, zwei Enzyme vorhanden sind, von denen das eine nur bei schwach saurer, das andre nur bei alkalischer Reaktion das Maximum seiner Wirksamkeit erreicht. Das amylolytische Enzym des Hafers und des Mais scheint durch anfängliche 6 stündige Einwirkung von $0,2 \%$ iger Salzsäure seine amylolytische Wirkung bei späterer alkalischer Reaktion nicht einzubüßen. Es kann also, selbst wenn es im Magen während längerer Zeit der Einwirkung der dort vorhandenen $\mathrm{HCl}$ ausgesetzt und zeitweise unwirksam war, im Darm unter den dort vorliegenden Verhältnissen wieder verdauend auf die Stärke der Nahrung wirken.

In bezug auf das Vorkommen eines Milchsäureenzyms konnten wir feststellen, daß ein solches in sämtlichen von uns untersuchten Nahrungsmitteln enthalten ist. Wir stellten je $1 \mathrm{~g}$ der Nahrungsmittel mit $50 \mathrm{~cm} \mathrm{H}_{2} \mathrm{O}$ bei $39-40^{\circ}$ in den Brütofen ein. Nach 2 und 6 Stunden wurde abfiltriert und im 
Filtrat mit $\mathrm{n} / 10-\mathrm{NaOH}$ der Säuregrad ermittelt, sowie mit Uffelmannschem Reagens auf Milchsäure geprüft. Nach 2stündigem Aufenthalte im Thermostaten war nur bei den Lupinenkörnern eine deutlich saure Reaktion nachzuweisen, nach 6 Stunden war aber bei sämtlichen Versuchen freie Milchsäure vorhanden. Nach dem Abfiltrieren gegen $\mathbf{n} / \mathbf{1 0}-\mathrm{NaOH}$ titriert ergab sich, berechnet auf die angewandte Substanz, folgender Milchsäuregehalt:

Pferdebohnen hatten gebildet: $0,36 \%$ Milchsäure

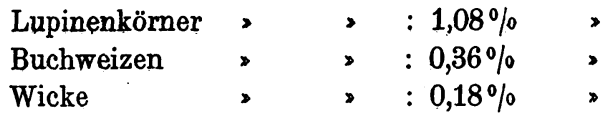

$\mathrm{Ob}$ der von uns festgestellte Säuregrad lediglich auf Rechnung der Milchsäure zu setzen ist, bleibt dahingestellt, jedenfalls konnte freie Milchsäure qualitativ stets nachgewiesen werden.

Ontersuchung auf ein in den Nahrungsmitteln vorhandepes proteolytisches Enzym.

Unsere Untersuchungen auf ein proteolytisches Enzym waren nur qualitativer Art und sollten nur feststellen, ob eine Peptonisierung der in den Nahrungsmitteln enthaltenen Eiweißkörper unter den im Magen und Darm herrschenden Bedingungen durch ein proteolytisches Nahrungsmittelenzym bewirkt werden kann.

Wir stellten zunächst fest, ob in den fraglichen Nahrungsmitteln Pepton vorhanden ist, indem wir je $5 \mathrm{~g}$ von fein geschrotenem Mais, Lupinenkörnern, Buchweizen, Wicken und Pferdebohnen mit $50 \mathrm{ccm}$ Wasser gut vermischt eine Viertelstunde lang im Eisschrank stehen ließen, hierauf filtrierten, im Filtrate die Eiweißkörper mit Essigsäure und Natriumsulfat in der Siedehitze entfernten und nach abermaligem Filtrieren und Auswaschen die Biuretreaktion im Filtrate anstellten. In keinem Falle konnte auf diese Weise eine Peptonreaktion erhalten werden.

Zum eigentlichen Versuche gelangten stets $5 \mathrm{~g}$ des betreffenden Nahrungsmittels mit $50 \mathrm{ccm} \mathrm{H}_{2} \mathrm{O}, 50 \mathrm{ccm} \mathrm{HCl}(0,2 \%)$ oder $50 \mathrm{ccm} \mathrm{Na}_{2} \mathrm{CO}_{3}(0,2 \%)$ auf 24 Stunden bei $39-40^{\circ}$ in den Thermostaten. Nach dieser Zeit wurden die Proben zunächst koliert und im Kolat die Eiweißkörper entfernt, deren Menge z. B. bei den Lupinen so groß war, daß nach ihrer 
Zur Kenntnis der in den Nahrungsmitteln enthaltenen Enzyme usw. 41

Abscheidung das ganze Verdauungsgemisch eine breiige Beschaffenheit zeigte. Im Filtrat wurde dann wieder die Biuretprobe angestellt. Die nachfolgende Tabelle enthält die Zusammenstellung der Resultate.

\begin{tabular}{|c|c|c|c|}
\hline $\begin{array}{c}\text { Art des } \\
\text { Nahrungsmittels }\end{array}$ & \multicolumn{3}{|c|}{$\begin{array}{l}\text { Peptonreaktion. } \\
\text { Die Verdauung ging vor sich in Lösung: } \\
\text { sauer } \mid \text { neutral } \mid \text { alkalisch }\end{array}$} \\
\hline Mais & negativ & $\begin{array}{l}\text { sebr schwach } \\
\text { positiv }\end{array}$ & $\begin{array}{c}\text { sehr schwach } \\
\text { positiv }\end{array}$ \\
\hline Pferdebohnen & $\begin{array}{l}\text { sehr stark } \\
\text { positiv }\end{array}$ & $\begin{array}{l}\text { sehr stark } \\
\text { positiv }\end{array}$ & schwach positiv \\
\hline Lupinen & negativ & $\begin{array}{c}\text { sehr stark } \\
\text { positiv }\end{array}$ & $\begin{array}{l}\text { sehr stark } \\
\text { positiv }\end{array}$ \\
\hline Buchweizen & $\begin{array}{l}\text { sehr stark } \\
\text { positiv }\end{array}$ & stark positiv & $\begin{array}{l}\text { sehr schwach } \\
\text { positiv }\end{array}$ \\
\hline Wicke & $\begin{array}{l}\text { sehr stark } \\
\text { positiv }\end{array}$ & $\begin{array}{l}\text { sehr stark } \\
\text { positiv }\end{array}$ & $\begin{array}{c}\text { sehr stark } \\
\text { positiv }\end{array}$ \\
\hline
\end{tabular}

Nach den vorstehend mitgeteilten Versuchsergebnissen erscheint es zweifellos feststehend, $d a B$ in allen untersuchten Nahrungsmitteln proteoly tis che Enzyme enthalten sind, die teils in alkalischer, teils in saurer Lösung das Maximum ihrer Wirksamkeit entfalten. Mais und Lupinenkörner enthalten nur in neutraler und schwach alkalischer Lösung wirkende proteolytische Enzyme, während bei Pferdebohnen und Buchweizen gerade die saure Reaktion des Verdauungsgemisches die Wirkung des Nahrungsmittelenzyms begünstigt. Bei Wicken konnten keine durch die Reaktion der Digestionsflïssigkeit bedingten Unterschiede in der Intensität der Peptonreaktion nicht festgelegt werden.

Für die Verdauungsvorgänge im Magen erscheint uns besonders wichtig, daß wenigstens bei einigen der Nahrungsmittel die Proteolyse nicht abhängig von der Reaktion des Mageninhaltes ist; trotzdem infolge der anfänglich im Magen der Pflanzenfresser herrschenden alkalischen, neutralen oder schwachsauren Reaktion (Milchsäure) eine peptische Wirkung 
ausgeschlossen ist, kann also die Eiweißverdauung mit Hilfe des proteolytischen Nahrungsmittelenzyms vor sich gehen. Dieser Befund kann vielleicht nicht allein für die Magenverdauung, sondern auch für die Darmverdauung von Wichtigkeit sein, indem im Darm die bei alkalischer Reaktion wirksamen Enzyme, welche durch die saure Reaktion des Mageninhaltes ihre Wirksamkeit noch nicht eingebüßt haben, den Eiweißabbau befördern. Unsre geringe Kenntnis von der Natur dieser Enzyme gestattet es nicht, weitere bindende Schlüsse zu ziehen, doch beabsichtigen wir, weitere quantitative Versuche anzustellen, um über Natur und Wirkungsweise des oder der in jedem Nahrungsmittel enthaltenen proteolytischen Enzyme Aufklärung zu erhalten.

Da uns der Enzymgehalt des Mais infolge unserer Arbeit über die Verdauung desselben beim Pferde ${ }^{1}$ ) besonders interessierte, wurde versucht, die Einwirkung verschiedener Säurekonzentrationen auf das nur bei schwach alkalischer und neutraler Reaktion wirkende Enzym kennen zu lernen. $\mathrm{Zu}$ diesem Zwecke gelangten je $5 \mathrm{~g}$ Mais mit $50 \mathrm{ccm}$ einer 0,2-, 0,1- und $0,05 \%$ igen $\mathrm{HCl}$ auf 24 Stunden in den Brütofen. Nach dieser Zeit hatte sich, wie zu erwarten, kein Pepton gebildet. Hierauf wurde genau neutralisiert und das Gemisch abermals 24 Stunden im Ofen belassen. Auch nach dieser Zeit konnte eine Peptonbildung nicht nachgewiesen werden. Es war also offenbar eine Zerstörung des proteolytischen Enzyms durch die in den oben genannten Konzentrationen einwirkende Salzsäure erfolgt. Eine Mitwirkung des proteolytischen Enzyms bei der bei alkalischer Reaktion ablaufenden Darmverdauung erscheint also bei Maisfütterung ausgeschlossen.

Bekanntlich herrscht aber in den ersten $1 / 3-2 / 3$ der Länge des Dünndarms längere Zeit nicht eine alkalische, sondern eine durch Milchsäure bedingte saure Reaktion. Hierbei ist eine proteolytische Mitwirkung des Maisenzyms nicht ausgeschlossen.

Untersuchungen auf ein celluloselösendes Enzym.

Mit der Frage der Celluloseverdauung im Blinddarm (siehe die vorige Abhandlung) beschäftigt, erschien es uns interessant,

1) Diese Zeitschrift, Bd. XLVII, S. 88. 
in den von uns untersuchten Futtermitteln auf ein cytohydrolytisches Enzym, eine Cytase, zu prüfen. Nach Brown ${ }^{1}$ ) soll ein derartiges Enzym im Verdauungstrakt, speziell im ersten Magen der Wiederkäuer vorhanden sein, und zwar kommt Brown auf Grund seiner mit Morris über die keimende Gerste ausgeführten Untersuchungen zu dieser Anschauung. Nach ihm ist die Cytase im Körnerfutter (auch im ruhenden Korn), speziell in Hafer und Gerste, präformiert vorhanden und gelangt dann in dem für ihre Wirksamkeit die günstigsten Bedingungen bietenden Vormagen (Pansen des Wiederkäuers) zur Tätigkeit.

Weiter nimmt Brown an, daß die gesamte Celluloseverdauung nur auf Grund dieses Nahrungsmittelenzyms vor sich gehe und andere Wirkungen, besonders die von Mikroorganismen, ausgeschlossen seien. $\mathrm{Da}$, wie in unserem Institute und von anderen Autoren nachgewiesen worden ist, die Celluloseverdauung im Tierkörper beträchtlich ist, müßten die fraglichen Körnerfrüchte demnach ein sehr kräftiges celluloselösendes Vermögen besitzen. Leider sind uns die Arbeiten Browns im Original nicht zugänglich gewesen, so daß sich kritische Betrachtungen von selbst verbieten. Es sei aber darauf hingewiesen, daß die Untersuchungsergebnisse Browns gerade in bezug auf das Vorkommen von Cytase in den Gramineen nicht ohne Widerspruch geblieben sind und daß sie durch die ausführliche Untersuchung Reinitzers ${ }^{2}$ ) in bezug auf das Vorkommen einer Cytase in der keimenden Gerste als widerlegt angesehen werden können.

Unsere diesbezüglichen Versuche wurden in folgender Anordnung ausgeführt. Zunächst wurde in den feingemahlenen Futtermitteln nach dem Verfahren von Henneberg und Stohmann (nacheinander folgendes Kochen von je $3 \mathrm{~g}$ mit $200 \mathrm{ccm}$ $1,25 \%$ iger $\mathrm{H}_{2} \mathrm{SO}_{4}$, Wasser, 1,25\% iger $\mathrm{KOH}$ und Wasser, Filtrieren durch gewogene Filter, Waschen, Trocknen, Wägen) der Rohfasergehalt ermittelt. Alsdann gelangten wiederum je $\mathbf{3}$ g mit $50 \mathrm{ccm}$ einer $0,2 \%$ igen Sodalösung auf 72 Stunden bei $38^{\circ}$ in den Thermostaten. Nach dieser Zeit wurde im Verdauungsgemisch wiederum nach derselben Methode der Roh-

i) J. chem. Soc., 1892, Bd. I, S. 352.

2) Diese Zeitschrift, Bd. XXIII, S. 175. 
fasergehalt ermittelt. Ein Vergleich der in $3 \mathrm{~g}$ enthaltenen aschefreien Rohfasermengen vor und nach dem Aufenthalt im Brütofen mußte, falls eine Verdauung von Cellulose stattgefunden hatte, dies durch eine Verminderung der Rohfasermenge anzeigen. Schwach alkalische Reaktion und 72 Stunden Digestionszeit wählten wir deshalb, weil dies den Verhältnissen am besten entspricht, unter denen die Celluloseverdauung im Dickdarm, speziell im Blinddarm, vor sich geht. Die Ergebnisse unserer Analysen finden sich in der folgenden Tabelle.

\begin{tabular}{l|c|c|c|c}
\hline & \multicolumn{4}{|c}{ Rohfasergehalt in Prozenten } \\
& Vor der Verdauung & \multicolumn{2}{|c}{ Nach der Verdauung } \\
& I. & II. & I. & II. \\
\hline Hafer . . . . & 14,60 & 14,57 & 14,60 & - \\
Pferdebohnen . & 6,57 & 6,65 & 6,48 & 6,67 \\
Lupinen . . . & 10,76 & 10,75 & 10,83 & 10,88 \\
Buchweizen . . & 11,53 & 11,45 & 11,45 & 11,55 \\
Wicke . . . & 4,45 & 4,35 & 4,58 & -
\end{tabular}

Der Vergleich der Resultate ergibt, daß in keinem Falle eine Verminderung der Cellulose eingetreten war und zwingt also zu dem Schlusse, daß ein celluloselösendes Enzym, eine Cytase, welche unter den im Tierkörper beim Ablauf der Celluloseverdauung gegebenen Bedingungen wirksam ist, in den untersuchten Nahrungsmitteln nicht enthalten ist.

\section{BohluBbetrachtung.}

Alle in unserem Institute ausgeführten Untersuchungen über die Nahrungsmittelenzyme bezweckten lediglich, die Mitwirkung dieser Enzyme bei den Verdauungsvorgängen in Magen und Darm festzustellen. Dabei sind folgende Ergebnisse gewonnen worden:

1. In vielen Nahrungmitteln: Hafer, Mais, rohen Kartoffeln, Reis, Erbsen, Gerste, Roggen, Weizen, Roggenstroh, Wiesenheu, Lupinenkörnern, Buchweizen, Wicken und Pferdebohnen befindet sich mindestens ein durch Siedehitze 
zerstörbares Enzym, welches bei Bluttemperatur imstande ist, die in den Nahrungsmitteln enthaltene Stärke zu Dextrinen und Zuckerarten abzubauen und dadurch löslich und für den Organismus verwertbar zu machen.

2. Durch Fütterungsversuche mit Tieren und durch Verdauungsversuche in vitro ist bewiesen worden, daß ein großer Teil der im Magen unserer Haustiere ablaufenden amylolytischen Vorgänge auf die Wirkung dieser Nahrungsmittelenzyme zurückzuführen ist.

3. Nach den vorstehenden Versuchen besitzen gewisse Nahrungsmittel amylolytische Enzyme, die ihre Wirkung nicht wie das Ptyalin des Speichels nur bei alkalischer, ganz schwach saurer und neutraler Reaktion zu entfalten vermögen, sondern vielmehr befähigt sind, Stärke auch bei einer relativ hohenSalzsäurekonzentration - 0,2\% - zu verzuckern, bei der das Speichelenzym längst unwirksam gemacht worden ist. Bei Wahl eines geeigneten Futtermittels findet demnach die Stärkeverdauung im Magen auch dann noch statt, wenn infolge der sauren Reaktion des Mageninhaltes das Ptyalin des Speichels seine Wirksamkeit längst eingebüßt hat.

4. Bei allen Versuchen geht mit dem Auftreten von Zucker auch das von freier Milchsäure Hand in Hand. Das Vorhandensein eines Milchsäurefermentes in oder an den Nahrungsmitteln ist demnach ebenfalls als erwiesen zu betrachten.

5. In den daraufhin untersuchten Nahrungsmitteln: Hafer, Mais, Pferdebohnen, Lupinen, Wicken und Buchweizen konnte das Vorhandensein mindestens eines proteolytischen Enzyms nachgewiesen werden, welches die in den Nahrungsmitteln enthaltenen Eiweißkörper bei Bluttemperatur zu peptonisieren vermag. Teilweise wirken diese Enzyme nur bei alkalischer oder saurer Reaktion, teilweise ist die Reaktion des Verdauungsgemisches auf sie bzw. ihre Wirkung ohne Einfluß. Im letzteren Falle vermögen sie demnach unter allen während der Verdauung im Magen herrschenden Reaktionsverhältnissen die Eiweißverdauung zu fördern. 6. Eincellulos elösendes, cy tohydroly tisches Enzym 
konnte in keinem der daraufhin untersuchten Nahrungsmittel von uns nachgewiesen werden.

7. Nicht nur bei der Magen-, sondern auch bei der Darmverdauung kommt die Mitwirkung der Nahrungsenzyme in Betracht; die meisten derselben können die Darmverdauung unter den im Darmkanal gegebenen Verhältnissen hochgradig fördern.

Die von Ellenberger erkannte große Wichtigkeit der Nahrungsmittelenzyme wird durch die vorstehenden Versuchsergebnisse von neuem bestätigt und es seien darum im nachfolgenden die Worte angeführt, die dieser Autor am Schlusse einer seiner ersten diesbezüglichen Publikationen ausspricht: ${ }^{1}$ )

Die Nahrungsmittelenzyme «haben eine praktisch wichtige Bedeutung für die Ernährung der Menschen und der Tiere bei Krankheiten der Verdauungsorgane. Bei geschwächter Verdauung ist die Verabreichung der pflanzlichen Nahrungsmittel in rohem Zustande der in gekochtem vorzuziehen. Es erklären sich hieraus zum Teil die Heilerfolge der Vegetarier und besonders der Körneresser bei Magenkatarrhen, Leberleiden u. dergl. Die roh eingeführten Nahrungsmittel machen im kranken Magen trotz der verringerten Menge an Verdauungssäften einen normalen Fermentations- resp. Verdauungsproze $B$ durch, der durch die in den rohen Nahrungsmitteln enthaltenen Fermente bedingt wird. Werden die Nahrungsmittel in gekochtem Zustande eingeführt, dann verfallen sie in den Verdauungsorganen abnormen Gärungen, deren Produkte Magenkatarrhe usw. zu verschlimmern geeignet sind. Durch das Kochen der Nahrungsmittel werden allerdings gewisse Nährstoffe löslicher gemacht, ja sogar gelöst, das Kochen ist also gewissermaßen eine Verdauung, damit ist aber für den Patienten nichts gewonnen. Erfahrungsgemäß treten bei Einführung von Dextrinen und Zuckerarten (wie sie beim Kochen und Backen stärkemehlhaltiger Nahrungsmittel entstehen) im Magen Gärungen viel leichter ein als bei Einführung des einfachen Stärkemehls.

1) Archiv f. wissenschaftl. u. prakt. Tierheilkunde, Bd. XIV, S. 62. 
Zucker und Dextrin werden von Personen mit schwacher Verdauung oft schlecht vertragen.

Zweifellos kommt bei den Körneressern, einer Sekte der Vegetarianer, noch in Betracht, daß dieselben die Körner im Munde gründlich durchkauen und einspeicheln, in einen milchartigen Brei umwandeln müssen, um dieselben gut schlingbar und schmackhaft zu machen. Die bedeutenden Speichelmengen, die infolgedessen während der Mahlzeit sezerniert und in den Magen eingeführt werden, wirken in doppelter Hinsicht günstig auf die Vorgänge im kranken Magen ein, indem einerseits die Alkalien des Speichels einen Teil der schädlichen Gärungssäuren binden, und indem andererseits das diastatische Ferment des Speichels in so bedeutenden Mengen in den Magen gelangt, daß es wesentliche Verdauungswirkungen daselbst entfalten kann. Auch die Wirkungsmöglichkeit des proteolytischen Fermentes der Körner wird durch den sezernierten Speichel gesteigert. Demnach empfiehlt es sich, den Menschen und Tieren, welche an Verdauungsschwäche infolge irgendwelcher Krankheiten leiden, gewisse vegetabilische Nahrungsmittel nicht in gekochtem, sondern in rohem Zustande und deshalb auch womöglich trocken zu verabreichen, damit dieselben tüchtig durchgekaut und eingespeichelt werden müssen.»

Diese Betrachtungen des genannten Autors beziehen sich fast nur auf die Vorgänge der Magenverdauung. Wir möchten aber nicht unterlassen, darauf hinzuweisen, daß sie auch für die Darmverdauung zum Teil gültig sind. Bekanntlich kommen Störungen der bei der Darmverdauung wirksamen Verdauungssekrete (z. B. Pankreasachylie) verminderte Gallen- und Darmsaftsekretion usw. nicht selten vor. Diese Störungen sind meist vergesellschaftet mit Magenleiden und nicht selten erst deren Folgen, es ist dann also auch die Absonderung des Magensaftes und damit nicht nur die Darm-, sondern auch die Magenverdauung gestört.

Eine Behandlung des Magenleidens durch Verabreichung von Diastase, Pankreaspräparaten usw. wirkt dabei oft günstig und heilend. Nach unserer Ansicht ist in solchen Fällen aber gerade die Ernährung der kranken Menschen mit rohen Nahrungs- 
mitteln (Körnern, rohen Früchten aller Art usw.) sehr am Platze. Die in diesen Nahrungsmitteln enthaltenen Enzyme treten an die Stelle der fehlenden Körperenzyme (der Enzyme des Pankreas-, Darm- und Magensaftes usw.) und bewirken einen nahezu normalen Verlauf der Verdauung. (Man wird dabei von der Verabreichung von Fleisch zeitweise am besten ganz absehen, obwohl im rohen Fleische auch Enzyme enthalten sind.) $\mathrm{DaB}$ mit einer solchen eigenartigen vegetarischen Ernährung gute Erfolge erzielt werden, wenn nicht unheilbare Organleiden vorliegen, hat die Erfahrung zur Genüge bewiesen. Die unter dem Einflusse der Nahrungsenzyme ablaufenden Verdauungsvorgänge genügen für die Ernährung des Kranken, bis die kranken Organe, die während dieser Zeit vor übermäßigen Funktionsreizungen geschützt sind, zur Norm zurückgekehrt sind.

Diese Darlegungen sollen nur zeigen, daß dieUntersuchungen auf das Vorhandensein von solchen Nahrungsmittelenzymen, die unter den im Magen und Darm gegebenen Verhältnissen wirken und die Verdauung wesentlich unterstützen und unter Umständen kompensatorisch für die krankhafterweise fehlenden oder in zu geringer Menge vorhandenen Körperenzyme eintreten können, nicht nur ein hohes wissenschaftliches, sondern auch ein praktisch-medizinisches Interesse haben. Es konnte aber nicht unsere Aufgabe sein, die letztere Frage weiter zu verfolgen. Für uns kam nur die physiologische Seite in Betracht, zu der wir im vorstehenden einen Beitrag geliefert haben.

\section{Nachschrift.}

Die Untersuchungen von Bergmann (Skand. Arch. für Phys., Bd. XVIII, S. 119) sind uns leider erst nach der Absendung dieser Arbeit an die Redaktion bekannt geworden, weshalb sie im vorstehenden eine Berücksichtigung nicht erfahren haben. 